ter where situated, showed the same clinical reaction, namely, swelling up at the menstrual period.

In my group of adenomyomas of the uterus were several of cervical origin. If these grow posteriorly. owing to their inherent tendency to become attached, they will spread out into the rectovaginal septum, and become adherent to the rectum; or the peritoneal surface of the cervix may grow fast to the peritoneal surface of the rectum. In either case the rectum becomes fixed to the cervix.

My Case 1 is probably the earliest stage of such a condition. Lying in the pelvic floor were several myomas, the smaller ones consisting of simple myomatous tissue, the largest one measuring 4 by 3 by $2 \mathrm{~cm}$. (Fig. 3 ) in diameter and containing a well-developed island of uterine mucosa (Fig. 4). It will be noted that even at this early stage the rectum was adherent to the posterior surface of the cervix. It was, however, possible to remove the uterus without injury to the bowel.

In Jessup's two cases the growth was so intimately blended with the rectum that in each it was necessary to remove a piece of the rectum with the uterus.

Lockyer's case represents a still further development of the adenomyoma. Such a wide area of the bowel was auherent that it was necessary to remove a complete segment of the rectum and to make a permanent colostomy.

In my Case 2, when the abdomen was opened, in San Francisco, the operator had found it necessary to remove a piece of the rectal wall with the adherent myomatous uterus. The patient's recovery was slow and complicated by an intestinal obstruction. When I saw the patient several years after the first operation she had had vaginal bleeding for months and was almost exsanguinated. When all attempts at building her up had failed, and the loss of blood still continued, as a last resort we did an exploratory operation, with the results recorded above. As noted from the history, the operation was necessarily an incomplete one, and hence it was impossible to tell the exact character of the thickening in the right broad ligament, but it was in all probability also adenomyomatous in character.

These cases are naturably of considerable interest to the surgeon. In the first place these growths, of course, are found only in women, and in the second place, histologically, they are not malignant; that is, they do not give rise to metastases. In the early stages, as shown in my Case 1, the growth may be removed without injury to the rectum. When the growth has invaded the rectum to a limited extent it is necessary to remove only a small portion of the anterior wall of the rectum and the defect can be closed, still leaving a bowel of sufficient caliber. When the rectal involvement is extensive, as in Lockyer's Case 2, resection of that portion of the bowel will, as a rule, be necessary.

The immediate differentiation between carcinoma of the bowel and adenomyomas of the rectovaginal septum is all-important to the surgeon. If the uterus contains myomas, the probability that the pelvic growth is an adenomyoma is strengthened. Further, if the growth appears to be muscular in origin this diagnosis is still more probable; if the growth is cystic, the diagnosis of adenomyoma is almost certain.

Cancer of the rectum starts in the mucous membrane, gradually infiltrates the bowel and then extends to the peritoneum and at a later stage may involve the cervix. Clinically, there is a history of hemorrhage from the bowel. In adenomyoma of the rectovaginal septum, on the other hand, the only rectal symptom is painful defecation, or there are obstructive symptoms. On rectal

examination the bowel mucosa may be found puckered but still intact. Thus it is seen that the differential diagnosis is relatively easy.

Adenomyomas of the rectovaginal septum are benign so far as they do not give rise to metastases, and consequently if the entire growth be removed no further trouble need be feared. If portions be left these will continue to grow and will lead to more pelvic adhesions, and finally produce complications that may result in death or permanent invalidism. When cancer of the rectum is present the operation must of necessity be a much more extensive one.

This group of cases still more clearly emphasizes the necessity of a careful microscopic control of all rectal growths, as they might easily pass for rectal carcinomas and the cases be classified as permanent cures of rectal cancer, when the patient in reality never had cancer.

In cases of adenomyoma of the rectovaginal septum the ureters should be carefully isolated and the uterus then removed in its entirety as in the Wertheim operation. As soon as the vagina has been cut across the uterus and rectum are more mobile and can be drawn further up into the wound. The necessary oval or elliptic piece of rectum is now removed with the uterus and the bowel wound closed. The remaining stages of the operation are identical with those of the Wertheim operation and it will be wișe to place a delicate protective drain in the pelvis, taking care, however, that it does not come in contact with the suture line in the bowel. The lower end of the drain emerges from the vagina.

When the adenomyoma involves a large part of the lumen of the bowel, it will become necessary to resect a segment of the bowel.

The glands in these growths undoubtedly arise from the uterine mucosa or from remnants of Müller's duct. If they were rectal adenomyomas, the glands would naturally be of the type composing the rectal mucosa.

The report of four cases in the course of a few months seems to indicate that adenomyoma of the rectovaginal septum is not rare; and with the more careful examination of all rectal or perirectal growths I feel confident that in the near future many more such cases will be recorded.

In conclusion I want to thank my friend, Mr. Max Brödel, director of the Department of "Art in Medicine," in the Johns Hopkins Medical School, for the excellent illustrations accompanying this article.

3 West Preston Street.

\section{DANGERS AT'TENDING INJECTIONS OF THE KIDNEY PELVIS FOR PYELOGRAPHY}

\author{
J. M. MASON, M.D. \\ BIRMINGHAM, ALA.
}

Although pyelography was introduced by Voelcker and Lichtenberg ${ }^{1}$ in 1906, its extensive employment in this country has been due, in large measure, to the work and writings of Braasch, of the Mayo clinic, in 1909 and subsequent thereto. Many shadow-casting substances have been tried, but collargol is the one which has been most generally used.

The beautiful results obtained in graphically depicting the shape and size of the kidney pelvis and ureter by means of pyelography, and the ease of its employment

1. Voelcker and Lichtenberg: München. med. Wchnschr., 1906 Cystographie und Pyelographie, Beitr. z. klin. Chir., Festschrift f.
Czerny, 1907. 
in connection with ureteral catheterization, toxether with its alleged freedom from danger, have led to its general employment as a diagnostic measure in kidney surgery.

Prior to 1913, in the reports from the Mayo clinic, $I$ have found no reference to any injurious effects on any of the kidneys subsequently subjected to operation,

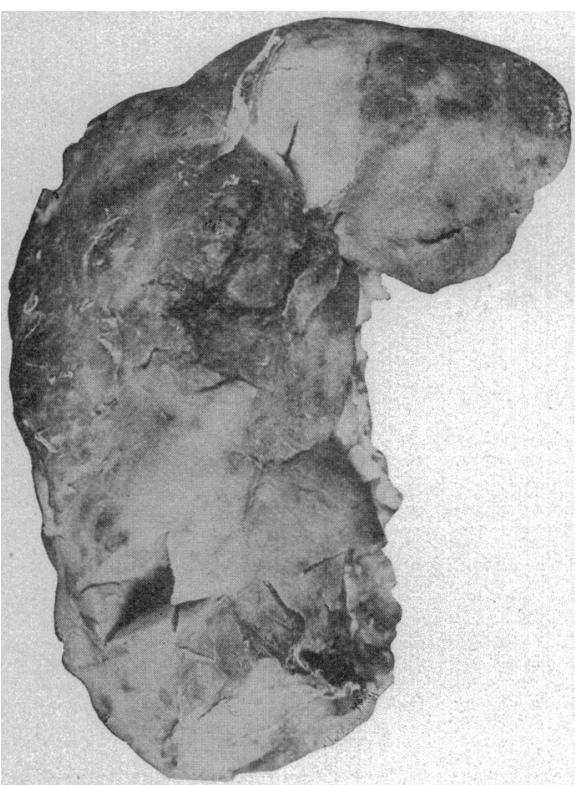

Fig. 1.-Outer surface of kidney in Case 1, showing discolored nodules on surface.

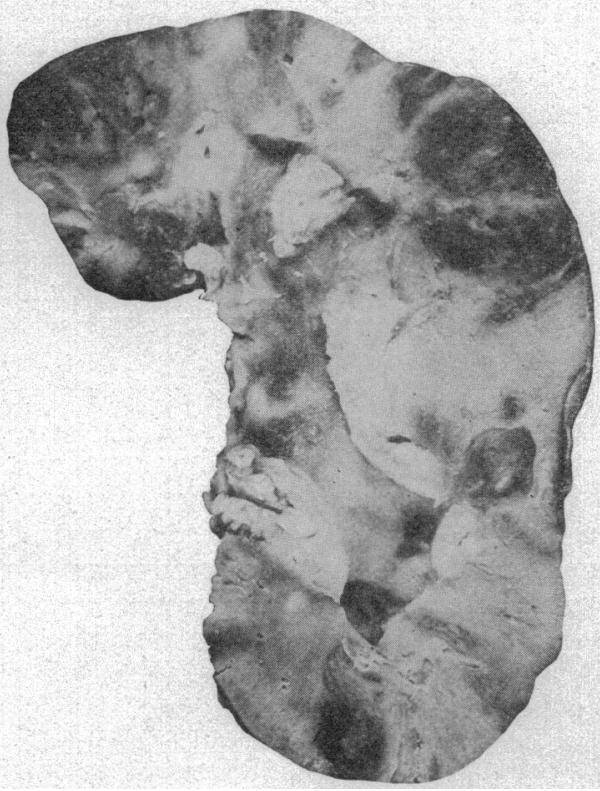

Fig. 2.- Section of kidney in Case 1, show ing collargol-stained infarcts. infarcts showing varying degrees of inflammation. No collargol remained in the pelvis.

CASE 2.-A few days later a patient with renal tumor, which proved to be a hypernephroma, came to me, and again collargol was injected.

Being mindful of the condition of the kidney following the injection in the former case, I was particularly careful with this injection, using 12 c.c. of 10 per cent. solution and stopping on the first appearanee of pain, and left the catheter in the ureter until I thought that all the collargol had run out. This injection was practically free from constitutional reaction. On removing the tumor ten days later, I found that this kidney also presented a few of the discolored nodules on its surface, and, on section, the same black-stained infarcted areas seen in the former case. The pelvis contained a quantity of precipitated collargol.

The following report of the microscopic examination of the infiltrated areas of the kidneys is submitted by Dr. E. M. Mason

"Irrespective of the primary pathologic condition in the kidney, one finds, in all sections, wide-spread deposits of collargol. Throughout the tubules collargolstained casts and granular deposits are numerous, and many of the malpighian corpuscles show collargol masses within the capsule of Bowman.

though Braasch ${ }^{2}$ states that he had frequently observed collargol retained in the kidney pelvis into which it had been injected several weeks before, and that its presence does not interfere with wound-healing. During 1913 reports from this clinic would seem to indicate some modification of the views previously entertained as to the innocuousness of injections into the kidney pelvis, and they will be referred to again.

In January, 1913, Thomas, ${ }^{3}$ of the Mayo clinic, writes:

The frequency of overdisten ${ }^{\perp}$ ion and renal colic resulting from injecting the pelvis of the kidney and ureters with shadow-casting fluids by means of a syringe, suggested to me the idea of using an apparatus with only sufficient forco of gravity to cause the flow to reach the kidneys.

With this gravity method, only ons colic resulted in more than fifty injections.

\section{REPORTS OF CASES}

CASE 1.--In February, 1913, there came into my hands a case of hydronephrosis due to ureteral stricture, in which I had occasion to inject the pelvis of the kidney with collargol.

Though I had often made use of pyelography without colic or constitutional disturbance, this patient suffered intensely, and the injection was rollowed by nausea and vomiting, and by a slight rise of temperature for four days. Twenty-five c.c. of 10 per cent. solution were injected before the slightest pain was expericnced.

When this kidney was removed five days later, its surface presented numerous nodular elevations showing black discoloration beneath the capsule. On section, each nodule proved to be the outer surface $o \hat{i}$ an infarcted area, cach infarct being deeply stained with coilargol, and the different

2. Rraasch, William F.: Recent Developments in Pyelography, Ann. Surg., 1010, lii, 645 .

3. Tliomas, G. J : An Apparatus for the Injection and Lavage of the Pelves of the Kidneys and Ureters, The JournaL A. M. A. Jan. 18. 1913 . p. 184
"No collargol is found in the blood-vessels or interstitial tissue of normal portions of the kidney, but the infarcted areas show large and small collargol masses both in the

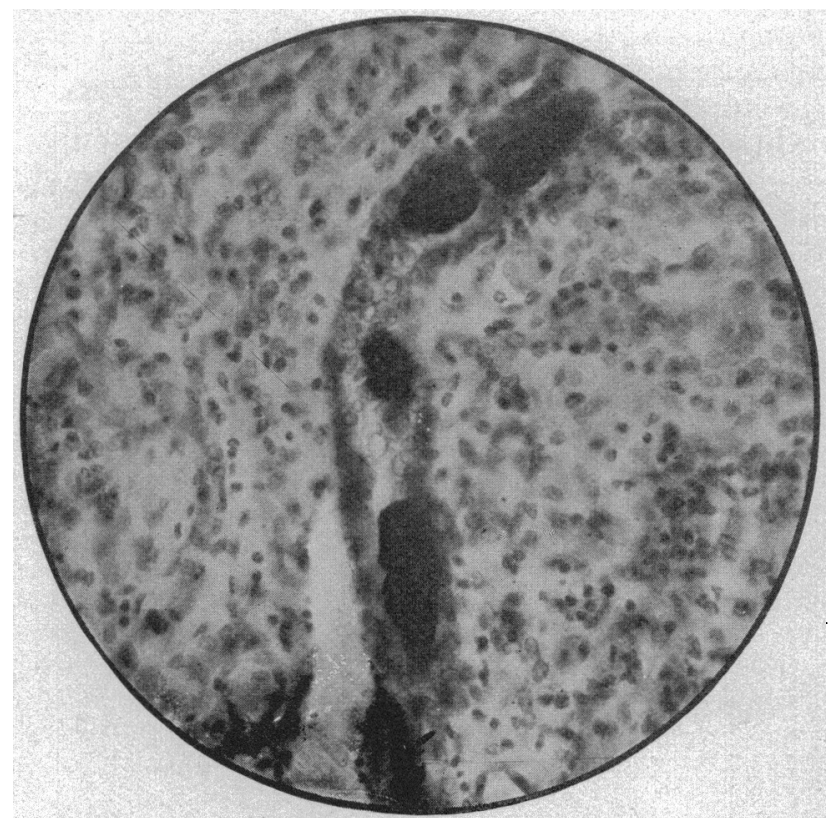

Fig. 3.-Collargol iying in the lumen of a straight tubule. Case $1 ; \times 230$

broken-down tubules and dispersed throughout the necrotic tissue as high up as the surface of the kidney.

"No definite evidence of inflammatory reaction can be seen in the collargol-containing tubules outside the necrotic areas, and there is no cellular infiltration of the adjacent interstitial tissue. 
"In some areas however, tube-casts, both stained and unstained, are more numerous than one would expect to find as a result of the primary pathologic condition."

Having considered up to this time, along with others, that the pain following injections into the pelvis was due to colic from overdistention, I was surprised at the condition of the kidneys in my cases, and set about

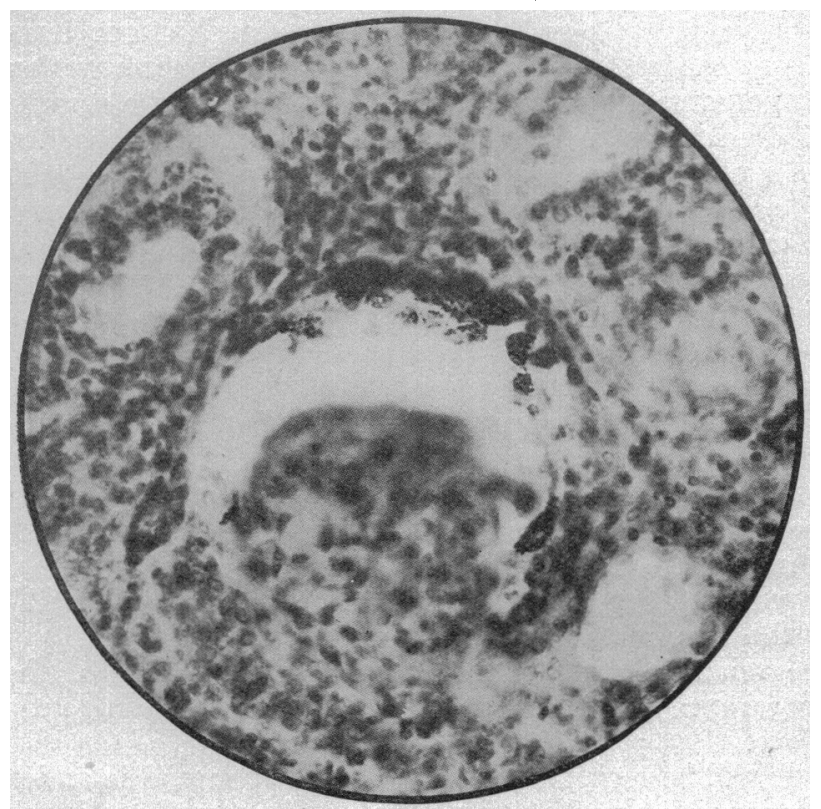

Fig. 4.-Collargol inside the capsule of a glomerulus, Case 1;
$\times 230$.

investigating the possible injurious effects of injections into the kidney pelvis.

In discussing my cases with Dr. G. S. Graham, professor of pathology in the Graduate School of Medicine of the University of Alabama, I learned that he had some sections of a kidney which he had seen in the laboratory of Prof. F. B. Mallory of Boston, which showed conditions similar to my own. Through his kindness I am able to present illustrations of these sections; and, through the kindness of Professor Mallory, I am able to give a brief description of the microscopic appearance of the kidney.

"Opposite hilum and toward one end, kidney presents a rounded elevation $4.5 \mathrm{~cm}$. in greatest diameter, dark red about periphery, with irregular, fine, slate-colored areas in central portion. Capsule has been torn away over part of this surface and is ragged and injected. Surface of kidney beneath is smooth. Section shows under this elevation an area roughly parallelogram in shape, extending $32 \mathrm{~mm}$. to the pelvis. Outer end is $25 \mathrm{~mm}$. wide and inner is $22 \mathrm{~mm}$. wide. This area consists roughly of three zones of about equal width; the inner is apparently the central portion of a pyramid showing striations, and is red in the center. Middle zone is a ring around this, is elevated and consists of yellowish-gray, pinhead areas, many of which are red points. The uneven junction between these two zones is dull slate-color with some dark-gray points. Outer zone is dull gray, and is interrupted by cortical tissue at medial distal corner. Smear from second zone shows polynuclear leukocytes but no tubercle bacilli or otner organisms. Frozen sections show black deposits."

Professor Mallory states that "the collargol was injected into the kidney tissue as a result of running the catheter too far into the pelvis of the kidney.

Evidently the point of the catheter went into a calix and the fluid was injected under pressure into that part of the kidney which drained into that calix."

He mentions having seen one other case in which the kidney was damaged by a collargol injection.

In 1913 Tennant reported a case which presented pathologic conditions in the kidney identical with those that I had observed.

Two injections were made into each kidney pelvis at intervals of five weeks. Pain followed each injection, particularly on the right side after the last injection.

Roentgenograms showed not only the outline of the kidney pelvis, but also a shadow well out in the parenchyma, extending from the center of the right kidney, and infiltrating its upper pole. This shadow was not understood or explained until operation two weeks later. when, after the kidney was freed from a great amount of inflammatory adhesions, an infarct 2 inches wide, and extending down to the renal pelvis, was exposed and resected. Microscopically, the same conditions were present as reported in my cases.

Kelly" says that in two cases in which he operated after collargol injection the perirenal tissues were stained a greenish black, the collargol having passed through the intact pelvis. In neither case was an excessive amount used, nor was it run in rapidly. He notes that one case required prolonged drainage.

Stover, in a foot-note to Tennant's paper, ${ }^{4}$ states that out of about forty instances of injections of the kidney pelvis under pressure, in early hydronephrosis due to ureteral obstruction, he had seen only two cases in which there was infiltration of the renal parenchyma.

While still insisting that ureteropyelography has been without fatality or permanent injury in his hands in more than one thousand cases, Braasch* states that instances have been reported by Buerger in which

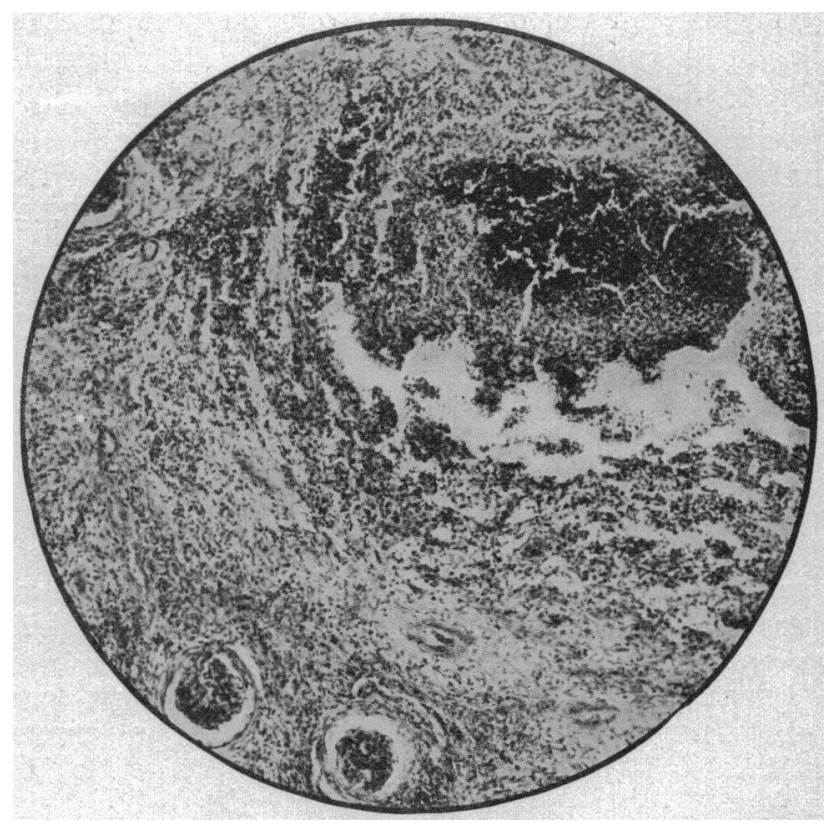

Fig. 5.-Collargol in the center of an abscess, Case $1 ; \times 60$.

deposits of silver, with surrounding foci of evident suppuration were found in the cortex of a kidney which was

4. Tennant: The Cause of Pain in Pyelography, with Report of Accident and Experimental Findings, Ann. Surg., 1913, Ivii, 888. 5. Kelly and Lewis: Silver Iodid Emulsion: A New Medium for Skiagraphy of the Urinary Tract, Surg., Gynec. and Obst., 1913, $x v i, 707$.

William F.: Recent Progress in Ureteropyelography, Jour. Michigan Med. Soc., April. 1913. No. 4. 
removed following pyelography, and that three similar cases have becn observed in the Mayo clinic. He also cbserved two cases of hydronephrosis injected with methylene-blue solution, which showed, at operation, cortical areas colored with the distending solution.

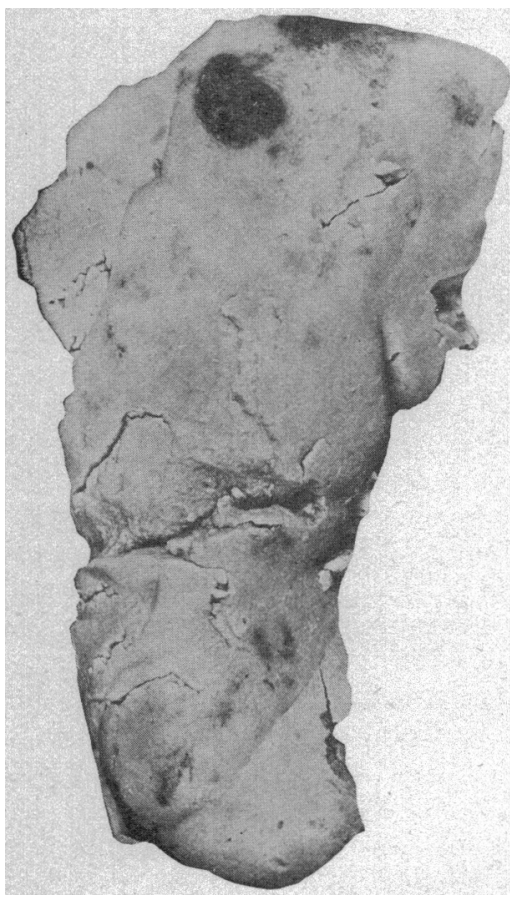

Fig. 6.-Outer surface of kidney in blackened nodules on the surface.

A. report by Zachrisien, ${ }^{8}$ in 1911 , of a case in which, by mistake, 14 c.c. of collargol solution were injected into the wrong kidney. This was followed by pain for five days, fever fourteen days, tenderness for some time, and the presence of casts in the urine from that side at the end of a year. The roentgenograms showed, as in Tennant's case, the outline of the pelvis, and also distinct dark shadows or streaks in the interior of the kidney, indicating that the injection had "certainly extended into the tubuli recti, and perhaps even higher."

A report by Roessle, ${ }^{9}$ in 1910 , of a fatality which he considered due to collargol poisoning, following an injection for pyelcgraphy; and, while this death might have been due to other causes, the kidney showed the presence of the injected collargol inside the tubules, and corresponded closely with the other cases reported.

Ekehorn, ${ }^{10}$ operating five days after collargol injection, found marked renal edema, and Jervell ${ }^{11}$ mentions observing a "wedge-shaped gangrenous area in the kidney" after the injection of collargol.

Experiments of Blum, Oehlecker and Tennant show results very similar to the observations on human beings.

Blum, ${ }^{12}$ using cadaver kidneys or extirpated kidneys, found that collargol passed through the ruptured col-

6. Ochlecker: Uebersichtsaurnahmen vom uro-poetischen Systeme (Pyelo-Kystographie), Fortsehr. a. d. Geb. d. Roentgenstrablen, 1911, xvi1, 195. urinkanaler och Malpighiska kroppar, Hyglea, Stockholm, 1913, Ixxv, No. 2 .

8. Zachrissen, 1911. Nord. Med. Ark, Abd. 1, No. 2.

9. Roessle: München. med. Wchnschr.. 1910, No. 5.

10. Kkrhorn, quoted by Key: Hygiea, 1911 , No. 2.

11. Jervell's case is referred to by Strassmann, Key, and Troell, but no record of its publication has been found.

12. Blum, Victor: Ueber den Werth der Pyelographie und anderer Methoden zum Nachweis von Dilatationen im Nierenbecken; Wien. med. Wchnschr., 1912, No. 19. lecting tubules, along the urinary canals and lymphspaces of the kidney, and under the capsule. He noted some instances of severe cauterizing effects on the mucosa of the kidney pelvis.

Oehlecker, ${ }^{6}$ using cadaver kidneys, found that collargol remained in the pelvis and in the calices, unless considerable pressure was used, when it was possible to force it into the tissues of the kidney.

Tennant, ${ }^{4}$ using fresh pig kidneys within one hour after removal from the animal, found that "infiltration of the parenchyma and free diffusion under the capsule of the kidney were almost simultaneous with the distention of the renal pelvis." This oceurred at once with 18 c.c. of collargol at a pressure of $240 \mathrm{~mm}$. of mercury, in one minute with 10 c.c. at a pressure of $10 \mathrm{~mm}$. of mercury, and in five minutes with 14 c.c. at a pressure of $50 \mathrm{~mm}$. of mercury. Sections of his kidneys showed the same micrescopic appearance as in his clinical case, except that no collargol was found in the capsules of the glomeruli. From these observations Tennant concludes that pain during injections of the kidney pelvis is not simply an indication of colic from overdistention of the pelvis, but that it indicates, or may indicate, a much more serious condition, namely, infiltration of the renal parenchyma with the injucted material. That this diffusion may take place simultaneously with the filling of the renal pelvis, and, presumably, at the time of the appearance of the pain, his experiments abundantly prove.

It will be seen that in all the cases presented in which the reports have been given in detail, as well as in all the experimental cases, the pathologic changes have been practically identical with my own; and we may safely infer that the gangrenous wedge-shaped area in Jervell's case belongs in the same group, and that the roentgeno-

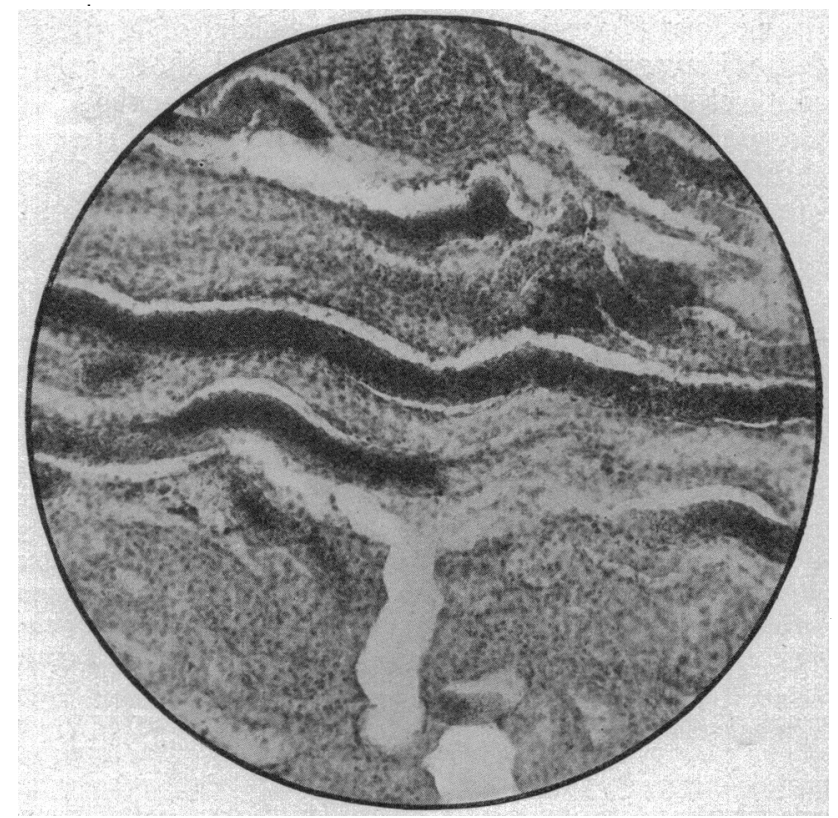

$\begin{array}{r}\text { Fig. } \\ \times 90 \\ \hline\end{array}$

grams in Zachrissen's case show that his patient's kidney received the same sort of injury.

By collecting these isolated clinical and experimental reports and comparing the findings, we are able to show, by the identity of the pathologic conditions, that a definite sequence of events follows injection of the renal 
pelvis if the intrarelvic pressure is raised beyond a certain unknown point.

This sequence comsists in the filling of the pelvis, and the ascent of the injected material, along with any infectious matter in the pelvis, into the straight and convoluted tubules and into the glomerular capsules. This may be followed by the rupture of the tuhnles with dissemination of their contents into the surrounding tissues, with the production of infarcts and abscesses, and progressive inflammation of the kidney and perirenal tissue.

The part that the collargol itself may play in the destructive changes is much in dispute. Some observers attribute severe cauterizing and irritating effects to this agent, others asserting that there is no injurious effect on the tubular epithelium or other parts of the kidney tissue. As collargol is the substance almost universally employed in the injections, we have no other drug or chemical used under like conditions of pressure in a series large enough for comparison.

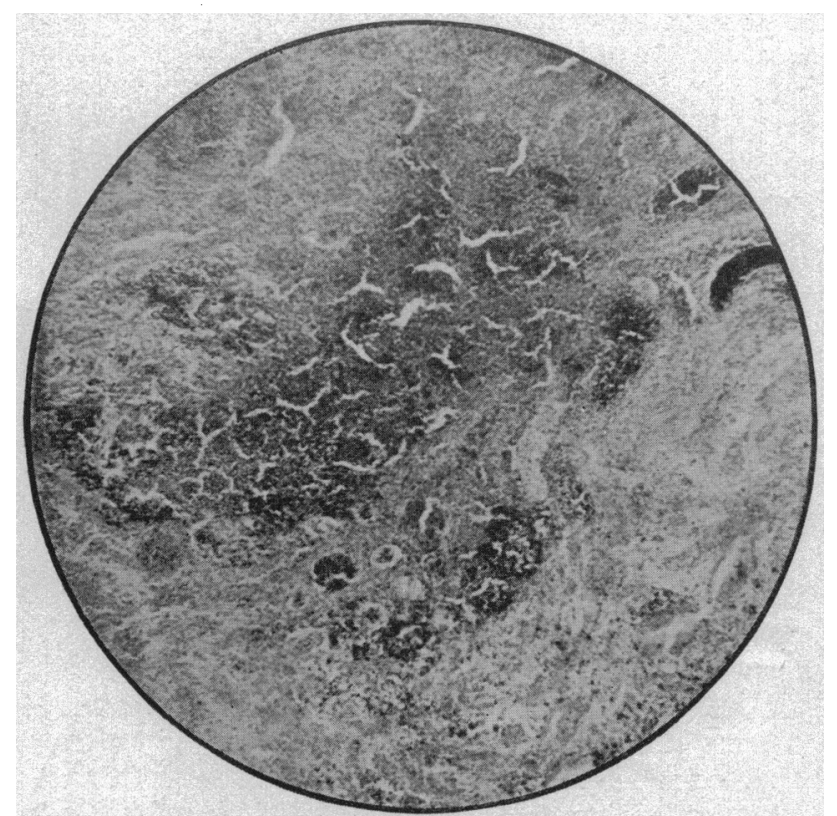

Fig. 8.-Collargol in tubule, Case 2 ; also broken-down kidneytissue with collargol disseminated throughout the tissue; $\times 60$.

Strassmann, ${ }^{\mathbf{1 3}}$ in 1913 , reviewed the question, and reached conclusions differing from the findings above set forth. $\mathrm{He}$ criticizes the experiments of Blum and Oehlecker, on the ground that there is too great difficulty in distinguishing between cadaveric changes and necrosis due to collargol, unless absolutely fresh material were used; also on the ground that Blum used too great pressure in his injections, and that the conditions surrounding his experiments were not normal and not comparable with experiments on the living. $\mathrm{He}$ thinks that conelusive experiments can be made only on living animals. He argues conclusively that the death which Roessle ascribed to collargol poisoning may have been due to some other cause. His explanation of the infarets observed by Oehlecker is that the tumor compressed the neck of the renal pelvis and interfered with the discharge of the collargol and thus forced it into the tubules.

13. Strassmann, Georg: Effect of Collargol Injections on the Kidney and Kidney Pelvis, Ztschr. f. Urol. Chir., 1913, i, Nos. 1 and 2 .
Together with Voelcker, he conducted a series of very delicate experiments on rabbits. Under anesthesia, the ureters were exposed and ligated, and the kidney pelves injected with collargol under the guidance of the eye, the amount of distention being easily seen. The fluid was retained in the pelvis for varying lengths of time, after which it was allowed to flow out and the kidney was removed for examination.

A summary of his observations is as follows:

From the renal pelvis occurs a rapid diffusion of the solution into the connective tissue surrounding the kidney. The remal capsule is swollen shortly after injertion and distinctly blackened in some portions. The injected collargol ascends, in part, into the connective tissue and lymphatic spaces of the kidney, particularly in the space around the urinary canals, and may ascend as far as the cortex. One may then see the urinary tubules surrounded by black masses. The intensity of discoloration of the capsule was directly proportionate to the time the collargol was retained in the pelvis.

The lumina of the tubules remained free. He states that, inasmuch as his injections were made under slight or only moderate pressure, the collargol advanced only as far as the renal pelvis. He asserts that there was no harmful effect on the pelvic mucosa, and that the silver masses surrounding the tubules exerted no injurions effects on the latter. He states that he used quantities of fluid in excess of the pelvic capacity, and that such quantities need not cause any injury.

$\mathrm{He}$ does not report the production of any infarcted areas; and, as his assertion is that the tubules remain free from the collargol and that the collargol ascends around the tubules, there could be no rupture of the tubules from pressure inside their lumen. His explanation of Oehlecker's infarcts mav have been correct in that instance, but does not hold good in other cases.

His results, at such variance from the data here presented, prove only that it is possible to inject the kidnev pelvis without damaging the parenchyma, an operation which has been done often enough for us to admit without question.

They do not prove that injections are free from danger, nor, in the light of the further evidence here presented, that the tubules and renal parenchyma may be expected to escape injury if the pressure within the pelvis is raised beyond a certain point.

It is scarcely possible to carry out, in man, an injection of the renal pelvis under the delicate control of pressure and pelvic distention that Strassmann exercised in his experiments, with the ureter and kidney in plain sight. We can only approximate his work clinically, by making injections with the utmost gentleness, and should avoid them if there is any interference with the rapid return flow of the injected material.

In an endeavor to account for the difference between Strassmann's pathologic observations and those of others, it has occurred to me that in his experiments he observed the process of normal absorption from the kidney pelvis of material present therein, but not under tension; whereas in the other cases the process was not one of passive absorption, but of active diffusion of the material under the influence of hydrostatic pressure inside the kidney pelvis.

This will explain, also, why so many injections have safely been made, and why such injuries as I have reported sometimes occur.

In his earlier writings Braasch advocates pyelography in practically cvery kidney disturbance requiring inves- 
tigation; in one paper enumerating no less than fifteen conditions in which it was indicated, including hydronephrosis, pyelitis, pyonephrosis and renal tubereulosis. ${ }^{2}$

In his papers for 1913, some modification of his former teachings is found, in that he states that it is contra-indicated in large hydronephroses, and in any case in which the pelvis cannot readily drain itself of the injected solution; that in malignant renal tumors it should not be used except when other means of diagnosis fail to identify the tumor ${ }^{14}$ that in his experience, severe reactions following pyelography have been due either to errors in technic or to lack of care in the selection of cases; and that pyelography should not be employed unless the existence or nature of a lesion in the urinary tract cannot be diagnosed in any other way.

To lessen the danger of overdistention, the gravity method of Thomas, or the still more accurate method of Tennant, who used a mercury manometer for estimating pressure, should be used. Even then the danger is not eliminated, for as yet we have no method of estimating the amount of pressure that may be safely used in

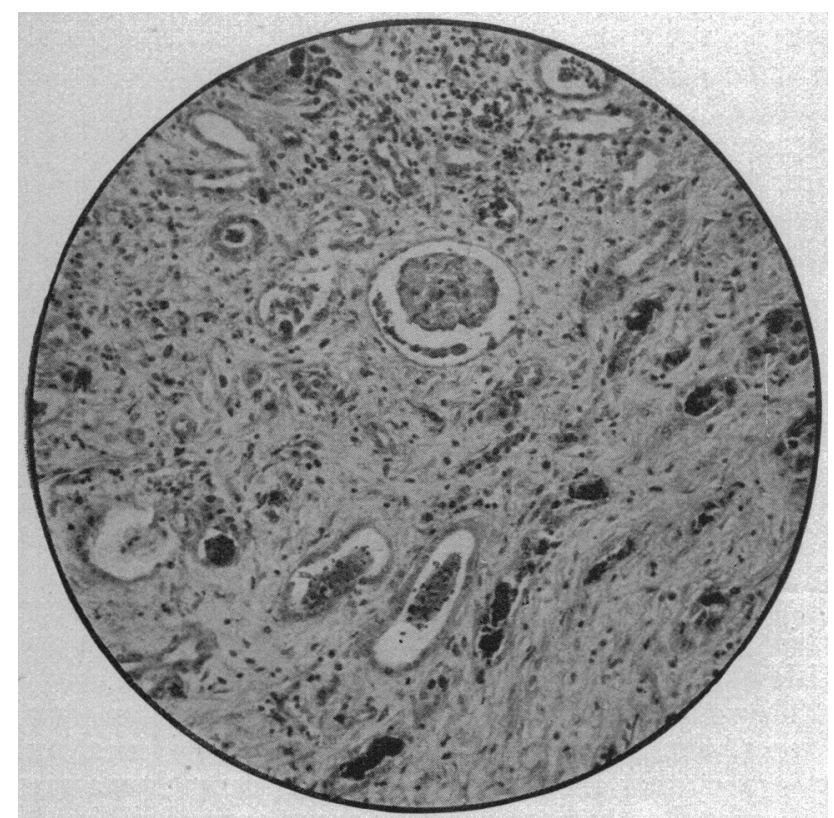

Fig. 9.-Section from Prof. Mallory's case; collargol in tubules and inside the capsule of a glomerulus; $\times 110$.

any given case; and, as this must vary in every case, a standard for comparison must be very difficult to establish.

The cases here presented prove that solutions injected into the kidney pelvis may, under circumstances which are not entirely under our control, ascend into the tubules and out into the surrounding kidney tissue carrying with them such infectious material as may be present in the pelvis and set up serious inflammatory lesions. With this clearly established, it would seem that injections are contra-indicated in the presence of infection in the kidney or kidney pelvis, and in cases in which the integrity of the kidney has been impaired by injury or disease.

14. Braasch Willium F.: Clinical Data on Malignant Renal Tumors, THE Joul XAL A. M. A., Jan. 25, 1913, p. 274.

Style.-Nothing is easier than to write so that no one can understand; just as, contrarily, nothing is more difficult than to express deep things in such a way that every one must necessarily grasp them.-Schopenhauer.

\section{RADIUM IN THE TREATMENT OF BLASTOMYCOSIS}

WITH REPORT OF A CASE

\section{FRANK EDWARD SIMPSON, M.D. \\ CHICAGO}

In a previous article ${ }^{1}$ the case now reported more fully was briefly referred to.

So far as I can learn, radium has not been used hitherto in the treatment of blastomycosis. A priori it

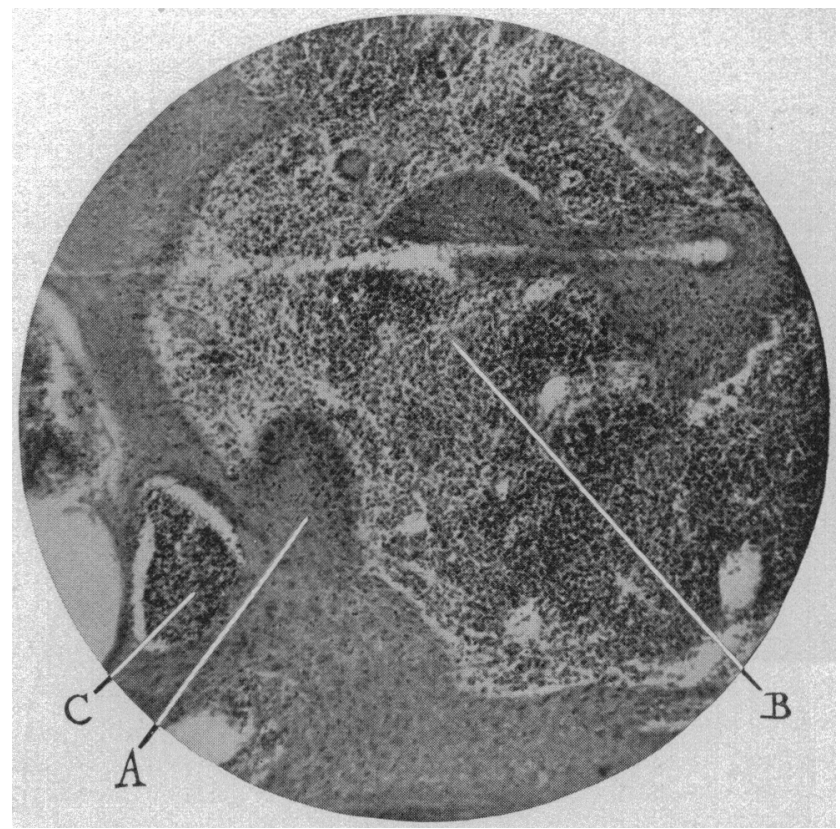

IIg. 1.-Transverse section showing proliferated epithelial lavers $(A)$ and on large $(B)$ and one small $(C)$ abscess; $X 90$.

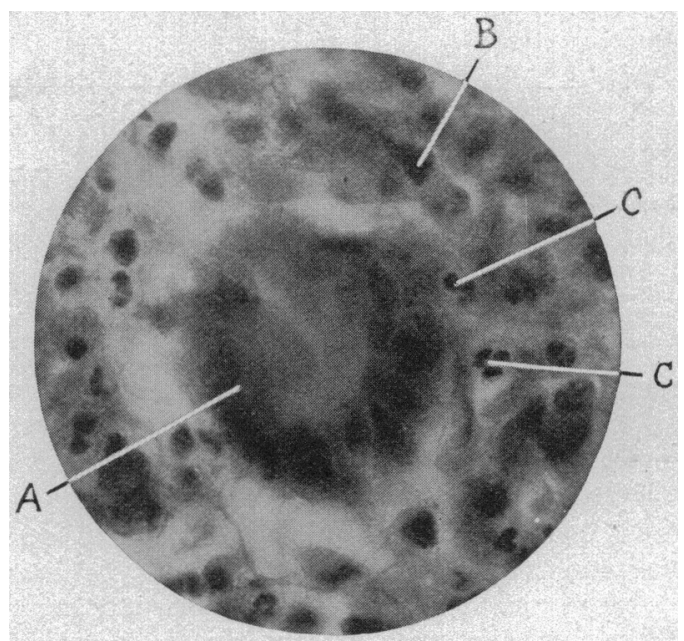

Fig. 2.-From same section. Multinuclear giant-cell (A) surrounded by epithelial cells $(B)$ and polynuclear leukocytes $(C)$; oil immersion; $\times 650$.

xas expected that radium would be effectual in this disease, and the opportunity of treating a case presented itself in May, 1913.

History.-The patient, a man, aged 24, came under observation at the Chicaro Policlinic Hospital with a lesion at the inner canthus of the left eye. The lesion, which was of three months' duration, involved the upper and the lower lid and covered an area of about $2 \mathrm{sq} . \mathrm{cm}$. (Fig. 3 ). It was of

1. Simpson, Frank E.: Radium in Skin Discases, The Jourxal A. 2. A., July 12, 1913, p. so. 